\title{
Influence of the Capping Agent PVP of the Outer Layer of Pd Nanocubes Surface on the Catalytic Hydrogenation of Unsaturated C-C Bonds
}

\author{
Suelen C. Buratto, ${ }^{a}$ Eloah Latocheski, ${ }^{a}$ Daniela C. de Oliveira ${ }^{b}$ and \\ Josiel B. Domingos ${ }^{\circledR} * a$ \\ ${ }^{a}$ Laboratório de Catálise Biomimética (LaCBio), Departamento de Química, \\ Universidade Federal de Santa Catarina, Campus Trindade, 88040-900 Florianópolis-SC, Brazil
}

${ }^{b}$ Laboratório Nacional de Luz Síncrotron, CP 6192, 13083-970 Campinas-SP, Brazil

\begin{abstract}
The excess of capping agents used during the synthesis of nanoparticles is usually removed by solvent washing. The influence of the amount of polyvinylpyrrolidone (PVP) capping agent in the catalytic activity of palladium nanocubes was evaluated, after different numbers of washing cycles in the purification step performed after the syntheses of the nanoparticles. The effect of PVP amount on the hydrogenation of unsaturated carbon-carbon bonds was evaluated in terms of kinetic analysis and diffusion experiments. Five different catalysts were prepared utilizing the washing off procedure. All catalysts were characterized by transmission electron microscopy, $\mathrm{X}$-ray photoelectron spectroscopy, small angle X-ray scattering spectroscopy and dynamic light scattering. The reaction presented strong influence by the amount of PVP, which was determined to be due to a balance between the amount of capping agent and the structure of the substrate.
\end{abstract}

Keywords: palladium nanocubes, capping agent effect, catalysis, hydrogenation, unsaturated $\mathrm{C}-\mathrm{C}$ bonds

\section{Introduction}

Polyvinylpyrrolidone (PVP), a non-toxic polymer which is soluble in many polar solvents, is the most used capping agent to stabilize catalytic metal nanoparticles synthesized by the colloidal methods. ${ }^{1}$ Its role on catalytic reactions depends on the synthetic conditions, but it is frequently argued that its presence on the surface of the nanoparticles is detrimental for the catalytic activity and thus its removal is a general consensus. ${ }^{2,3}$ Several methods has been proposed in the literature ${ }^{3-8}$ to effectively remove PVP from the surface of the nanoparticles, including thermal treatment, chemical reduction, oxidative, electrochemical and plasma treatment, and solvent extraction, among other approaches. Nevertheless, no work has been preoccupied with the fact that the frequently applied post-synthesis purification step, the washing off procedure of PVP excess, may lead to a variable amount of the stabilizer on the nanoparticles surface and that by itself may affect the catalytic activity of the nanoparticles. Thus, we report herein an investigation on the effect of PVP excess, remained on the purification step of palladium nanoparticles synthesis, in the catalytic

*e-mail: josiel.domingos@ufsc.br efficiency on the hydrogenation of unsaturated carboncarbon bonds.

PVP is able to interact with the surface of the nanoparticles through multiple types of contact, by chemisorbing with both the oxygen atom and nitrogen atom in the ring. ${ }^{9}$ Part of the PVP adsorbs on the first capping layer, while the other part dissolves in the suspension, creating capping/protective outer layers (Scheme 1) and these PVP outer layers are the ones which are washed off in the standard nanoparticles purification protocols.

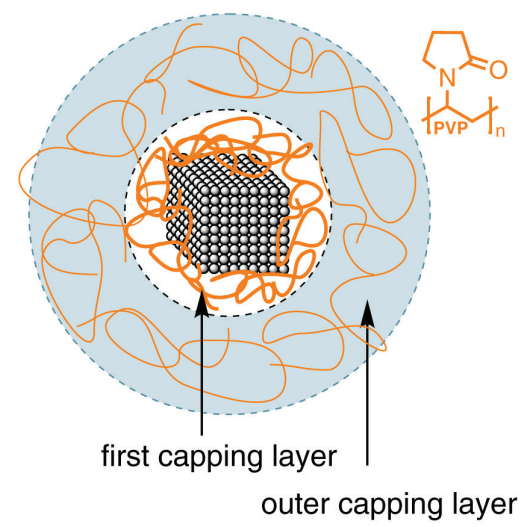

Scheme 1. Conformation model of PVP-stabilization of metal nanoparticles. 
In the present work, the influence of the capping agent (PVP) remaining on palladium nanoparticles after the purification procedure, in terms of their catalytic activity, was evaluated on the hydrogenation of unsaturated carbon-carbon bonds, through kinetic analysis and diffusion experiments.

\section{Experimental}

\section{Materials and methods}

The polyvinylpyrrolidone (PVP, $55 \mathrm{kDa}$ ), $\mathrm{K}_{2} \mathrm{PdCl}_{4}$, L-ascorbic acid (AA), styrene (ST), phenylacetylene (PA), stilbene (STB), diphenylacetylene (DPA) and potassium bromide were purchased from Sigma-Aldrich (São Paulo, Brazil) and used as received without any further purification. All solvents were spectroscopic grade and ultrapure water (TKA Smart2Pure, Niederelbert, Germany) was used in all systems.

Synthesis and washing procedure of palladium nanocubes (Pd-NCs)

The synthesis of palladium nanocubes (Pd-NCs) was performed as previously described in the literature. ${ }^{10}$ In a two-necked round bottom flask, $105 \mathrm{mg}$ PVP, $60 \mathrm{mg}$ AA and $300 \mathrm{mg} \mathrm{KBr}$ were dissolved in $8 \mathrm{~mL}$ of water and stirred for $5 \mathrm{~min}$ at $80^{\circ} \mathrm{C}$. In the next step, $65 \mathrm{mg}$ of $\mathrm{K}_{2} \mathrm{PdCl}_{4}$ were added in $3 \mathrm{~mL}$ of water and the mixture was aged for $4 \mathrm{~h}$ at $80{ }^{\circ} \mathrm{C}$. The resulting black solutions were mixed with acetone and centrifuged. To control the amount of PVP, additional washing steps were performed 1 to 8 times, as follows. The precipitated NCs were dispersed in $500 \mu \mathrm{L}$ ethanol and precipitated by addition of $35 \mathrm{~mL}$ acetone, the $\mathrm{NCs}$ in powder form were recovered by centrifugation at $800 \mathrm{rpm}$ for $20 \mathrm{~min}$. The amount of PVP on the surface of the NCs was established by the difference between the total mass and the mass of the metal determined by flame atomic absorption spectrophotometry (FAAS) (Table S1, Supplementary Information (SI) section). The method proved to be effective, ranging from $85(1 \times)$ to $20 \%(8 \times)$ in the total amount of PVP $\left(\mathrm{m} \mathrm{m}^{-1}\right)$.

\section{Characterization of $\mathrm{Pd}-\mathrm{NCs}$}

All NCs were characterized by transmission electron microscopy (TEM), small-angle X-ray scattering (SAXS), dynamic light scattering (DLS) and X-ray photoelectron spectroscopy (XPS). For the TEM analysis, a sample of Pd-NCs was dispersed in water and dropped onto a 200 mesh copper grid with carbon film and dried naturally. The experiments were conducted on a JEOL JEM-1011 microscope operating at $100 \mathrm{kV}$. SAXS experiments were performed on the SAXS1 beamline of the Brazilian Synchrotron Light Laboratory (LNLS, Campinas, SP, Brazil). The solutions were loaded into a temperaturecontrolled vacuum flow-through cell composed of two mica windows separated by $1 \mathrm{~mm}$, normal to the beam. ${ }^{11}$ The collimated beam $(\lambda=1.55 \AA)$ crossed the sample through an evacuated flight tube and was scattered to a Pilatus $300 \mathrm{~K}$ 2D detector (Dectris). The incident beam was detected at sample to detector distances of 500 and $3000 \mathrm{~mm}$ (silver behenate was used for the sample to detector distance calibration). In all cases the $2 \mathrm{D}$ images were found to be isotropic and they were normalized using the FIT2D software developed by Hammersley. ${ }^{12}$ In addition, the resulting scattered intensity $\mathrm{I}(\mathrm{q}) v s$. scattering vector $\mathrm{q}$ curves were corrected by subtraction of the scattering curve from the pure solvent curve and then placed on an absolute scale using water as the standard. The I(q) $v s$. q scattering profile of the NCs could be fitted using the form factor of homogeneous spheres with a power-law regime to adjust the background. The fitting procedures and other analyses were performed using the SASfit software,${ }^{13}$ which makes use of the least-squares fitting approach to minimize the $\chi^{2}$ parameter. The SASfit software package was developed by Kohlbrecher and is available free of charge. ${ }^{13}$ The XPS data for the samples were obtained with an SPECSLAB II (Phoibos-Hsa 3500 150, 9 channeltrons) spectrometer using a X-ray source $\mathrm{Al} \mathrm{K} \alpha$ which provide photons with $1486.6 \mathrm{eV}$ on Brazilian Synchrotron Light Laboratory (LNLS, Campinas, SP, Brazil). The acquisition parameters were pass energy (Epass) of $40 \mathrm{eV}$, energy step of $0.1 \mathrm{eV}$ and acquisition time of $1 \mathrm{~s}$ per point. The samples were placed on stainless steel sample holders and transferred to the XPS analysis chamber where the internal residual pressure was around $1 \times 10^{-8}$ Torr. Faster scans were performed in $\mathrm{Pd}$ region to prevent radiation and vacuum damage on samples. The binding energies (BE) of Pd $3 d$, $\mathrm{N} 1 \mathrm{~s}, \mathrm{C} 1 \mathrm{~s}$ and $\mathrm{O} 1 \mathrm{~s}$ were referenced to the Si $2 \mathrm{p}$ peak at $104.5 \mathrm{eV}$, providing accuracy within $\pm 0.2 \mathrm{eV}$. The DLS experiments were performed using a Zetasizer Nano ZS instrument (Malvern Instruments, UK), the samples were measured at a constant temperature of $25 \pm 1{ }^{\circ} \mathrm{C}$. The FAAS analysis was carried out with a Hitachi Z-8230 Polarized Zeeman spectrometer. The water phase containing the Pd-NCs was centrifuged and the supernatant was mixed with $10 \%(\mathrm{v} / \mathrm{v})$ of an aqua regia solution for the measurements.

\section{Catalytic activity studies}

All catalysts were tested for the hydrogenation of styrene, phenylacetylene and diphenylacetylene. The 
reactions were performed in a Fischer-Porter type glass reactor with instant $\mathrm{H}_{2}$ level monitoring under stirring and pressure of 4 bar at a temperature of $60{ }^{\circ} \mathrm{C}$. The substrates were added at the concentration of $0.5 \mathrm{~mol} \mathrm{~L}^{-1}$ in tetrahydrofuran (THF) and $0.5 \mathrm{~mol} \%$ of catalyst in the reactor. The amount of catalyst was defined by the total concentration of metal in the sample determined by FAAS. The reactions were monitored until the hydrogen consumption stabilized. After the reaction, the reactor was left to reach room temperature, the $\mathrm{H}_{2}$ pressure was removed, and an aliquot was taken for GC-MS (gas chromatography coupled with a mass spectrometer) analysis.

\section{Gas chromatography analysis}

The crude solutions obtained after catalytic experiments were filtered using a small plug of silica and each obtained filtrate was injected into GC-MS for analysis and determination of hydrogenation conversion. The GC analysis were run with an Agilent Technologies 7820A instrument coupled to Agilent Technologies 5975 mass spectrometer, equipped with an HP5 chromatography column (Agilent, $30 \mathrm{~m} \times 0.25 \mathrm{~mm} \times 0.25 \mu \mathrm{m}$ ), in isobaric conditions at $60 \mathrm{kPa}$, using helium as the carrier gas $\left(1 \mathrm{~mL} \mathrm{~min}^{-1}\right)$, the temperature of the injector was set at $250{ }^{\circ} \mathrm{C}$ and the temperature of the column set at $60^{\circ} \mathrm{C}$ (3 min). Initially, each analysis was performed using a temperature program from 60 to $150{ }^{\circ} \mathrm{C}\left(5^{\circ} \mathrm{C} \mathrm{min}-1\right)$ and then another from 150 to $280^{\circ} \mathrm{C}\left(20^{\circ} \mathrm{C} \mathrm{min}^{-1}\right)$. Conversions were determined by comparing the areas of product and reagent peaks.

\section{Results and Discussion}

To evaluate the effect of the capping agent PVP left over on palladium nanoparticles after a purification procedure, we chose to use palladium nanocubes (Pd-NCs), for the fact that their possible shape modification induced by the washing procedure is readily assessed by standard analytical tools, as TEM analysis. The washing protocol applied is the one normally reported on the literature. By simply dispersing the Pd-NCs in ethanol and inducing its precipitation with acetone, by polarity/solubility control (or number of repetitions), we could manage to vary the amount of PVP on the Pd-NCs from 85 to $20 \%\left(\mathrm{~m} \mathrm{~m}^{-1}\right)$ (named Pd-NC85, Pd-NC70, Pd-NC55, Pd-NC39 and Pd-NC20, according to the \% amount of PVP left on the Pd-NCs after the washing procedure, Table S1, SI section) in a very proportional manner and with high degree of reproducibility (Figure 1).

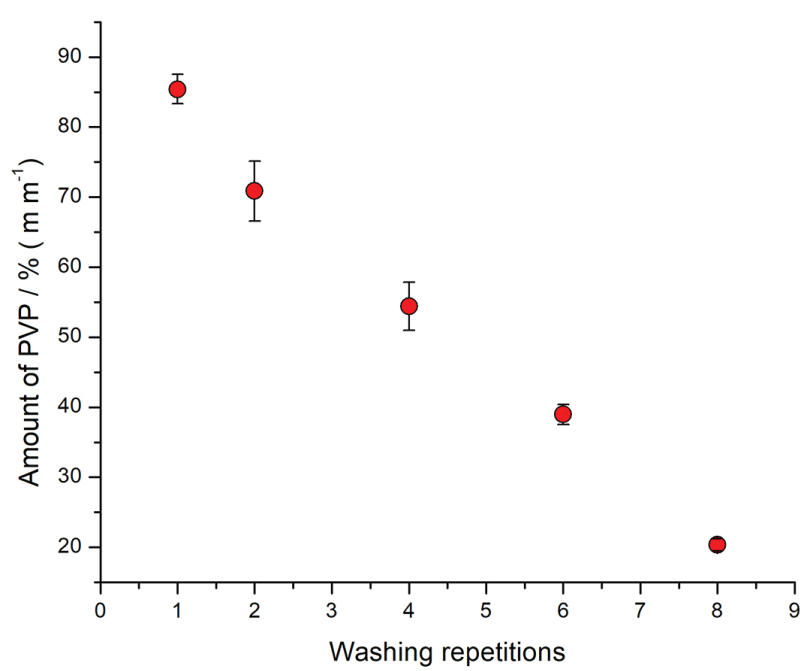

Figure 1. Amount of PVP remained on the surface of Pd-NCs as a function of washing repetitions.

After every washing repetition, samples were collected and carefully analyzed by different analytical tools. TEM was used to determine the edge length, shape and size distribution of the Pd-NCs (Figure 2). Two perpendicular edges of each cube were measured and plotted versus each other to confirm the cubic morphology (Figure 2f). The average size histograms showed a Gaussian distribution and confirmed that the washing off procedure did not affected the nanocubes $(\mathrm{NCs})$ mean diameter, with $11.5 \pm 1.9$, $11.8 \pm 1.6,11.8 \pm 1.6,11.3 \pm 1.4$ and $11.5 \pm 1.9 \mathrm{~nm}$ for Pd-NC85, Pd-NC70, Pd-NC55, Pd-NC39 and Pd-NC20, respectively. However, it is possible to see a higher degree of agglomeration with the increase of washing times. This is probably due to a smaller steric repulsion with the reduction of PVP concentration on the outer layers of the Pd-NCs.

Synchrotron-based SAXS measurements were also performed to confirm the size and dispersity $(\sigma)$ of the NCs. Figure 3a shows the SAXS profile for Pd-NC85, which was refined according to a spherical form factor (see Figure S1, SI section, for all catalysts). ${ }^{14,15}$ The radius of gyration, $R_{G}$, can be obtained at the low limit of $q$, according to the Guinier approximations. The $\mathrm{R}_{\mathrm{G}}$ values for a dense sphere and a perfect cube are given by equations 1 and 2 , respectively:

$\mathrm{R}_{\mathrm{G}}^{2}=\frac{3}{5} \mathrm{R}_{\mathrm{S}}^{2}$

$\mathrm{R}_{\mathrm{G}}^{2}=\frac{1}{4} \mathrm{e}_{\mathrm{c}}^{2}$

where $R_{S}$ is the sphere radius and $e_{c}$ is the cube edge length. Thus, the edge length of the cube can be calculated by the sphere radius, using equation 3 : 


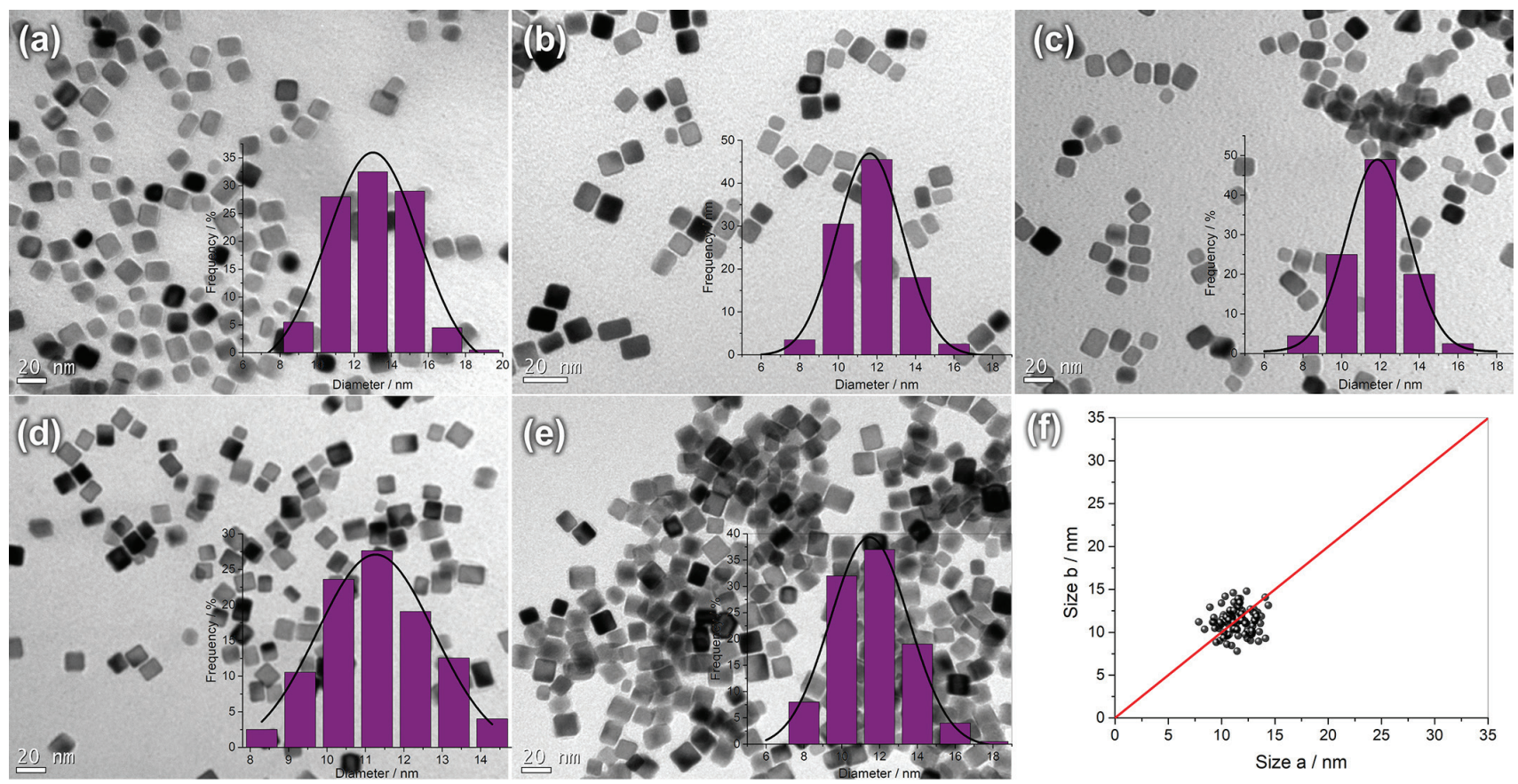

Figure 2. TEM micrographs of the palladium nanocubes varying the amount of PVP after every washing repetition procedure: (a) 85\% (Pd-NC85, mean diameter $(\mathrm{dm})=11.5 \pm 1.9 \mathrm{~nm})$; (b) $70 \%(\mathrm{Pd}-\mathrm{NC} 70, \mathrm{dm}=11.8 \pm 1.6 \mathrm{~nm})$; (c) $55 \%(\mathrm{Pd}-\mathrm{NC} 55, \mathrm{dm}=11.8 \pm 1.6 \mathrm{~nm}) ;(\mathrm{d}) 39 \%(\mathrm{Pd}-\mathrm{NC} 39, \mathrm{dm}=11.3 \pm 1.4 \mathrm{~nm})$; (e) $20 \%$ (Pd-NC20, dm = $11.5 \pm 1.9 \mathrm{~nm}$ ); and (f) side a vs. side b plot.
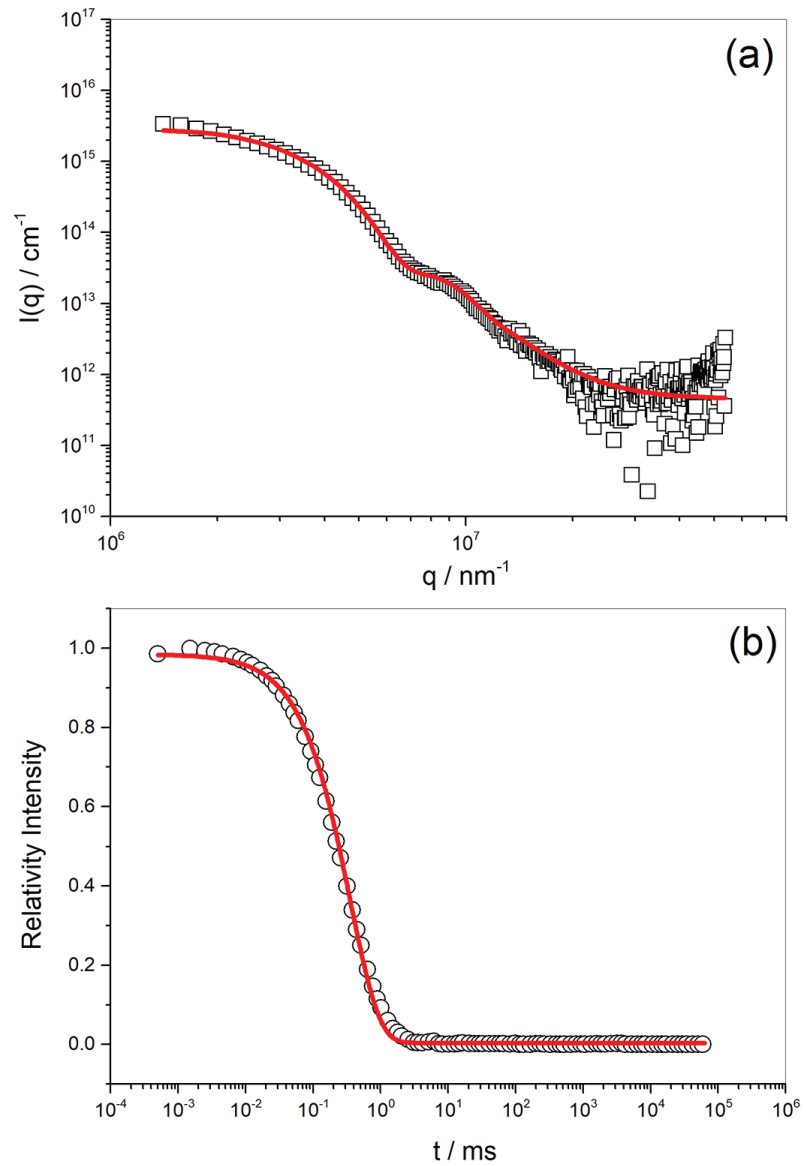

Figure 3. (a) SAXS data and corresponding curve fitting; (b) volumeweighted DLS $\left(D_{H}=120.8 \mathrm{~nm}\right)$ for Pd-NCs with $85 \%\left(\mathrm{~m} \mathrm{~m}^{-1}\right)$ of PVP on the surface.

$$
\mathrm{e}_{\mathrm{c}}=\sqrt{\frac{12}{5}} \mathrm{R}_{\mathrm{S}}
$$

The calculated $\mathrm{R}_{\mathrm{G}}, \mathrm{R}_{\mathrm{S}}, \mathrm{e}_{\mathrm{c}}$ and $\sigma$ values are presented on Table S2 (SI section) for all Pd-NCs. These data agreed with the results obtained by TEM, showing that the mean size of the NCs were not affected by the purification procedure, and thus a parameter that does not need to be considered for the comparison of the catalytic activities.

Additional characterization of the Pd-NCs with light scattering technique (DLS) was performed. The correlation curve of the dynamic light scattering for Pd-NC85 is shown in Figure 3b (see Figure S2, SI section, for the other NCs). The average hydrodynamic diameters $\left(\mathrm{D}_{\mathrm{H}}\right)$ of the Pd-NCs were determined to be 120.8, 97.9, 163.7, 158.6 and $96.8 \mathrm{~nm}$ for Pd-NC85, Pd-NC70, Pd-NC55, Pd-NC39 and Pd-NC20, respectively. Nevertheless, the intensity of light scattering was heavily weighted by the particle molecular weight. Therefore, by considering the number of particles, the presence of large aggregates can be neglected as clearly evidenced by the TEM micrographs.

XPS analysis was performed on samples to identify what species are present at the surface of particles with different PVP contents. Figure 4 shows Pd 3d region for each sample. Fitting procedure was done by using asymmetric line shapes for metallic $\mathrm{Pd}\left(\mathrm{Pd}^{0}\right)$ and symmetric for oxidized species $\left(\mathrm{Pd}^{\delta+}\right)$. On Pd-NC85 and Pd-NC70 samples we found only oxidized species, according to the 
binding energies (BE) of Pd 3d 5/2 core-levels (Table 1). ${ }^{16}$ This demonstrates that oxidized Pd is found on the surface of nanoparticles. $\mathrm{Pd}^{0}$ is identified only after few washes, being observed on Pd-NC55, Pd-NC39 and Pd-NC20 samples. This is related to the higher concentration of $\mathrm{PVP}$ in the Pd-NC85 and Pd-NC70 samples that block the more internal $\mathrm{Pd}^{0}$ electrons to be detected. The better signal-tonoise quality of spectrum from the more washed samples also corroborates the idea of the various layers of PVP in the NC being removed and more Pd being exposed. The $\mathrm{Pd}^{0} / \mathrm{Pd}^{\delta+}$ ratios calculated from the XPS spectra (Table 1)
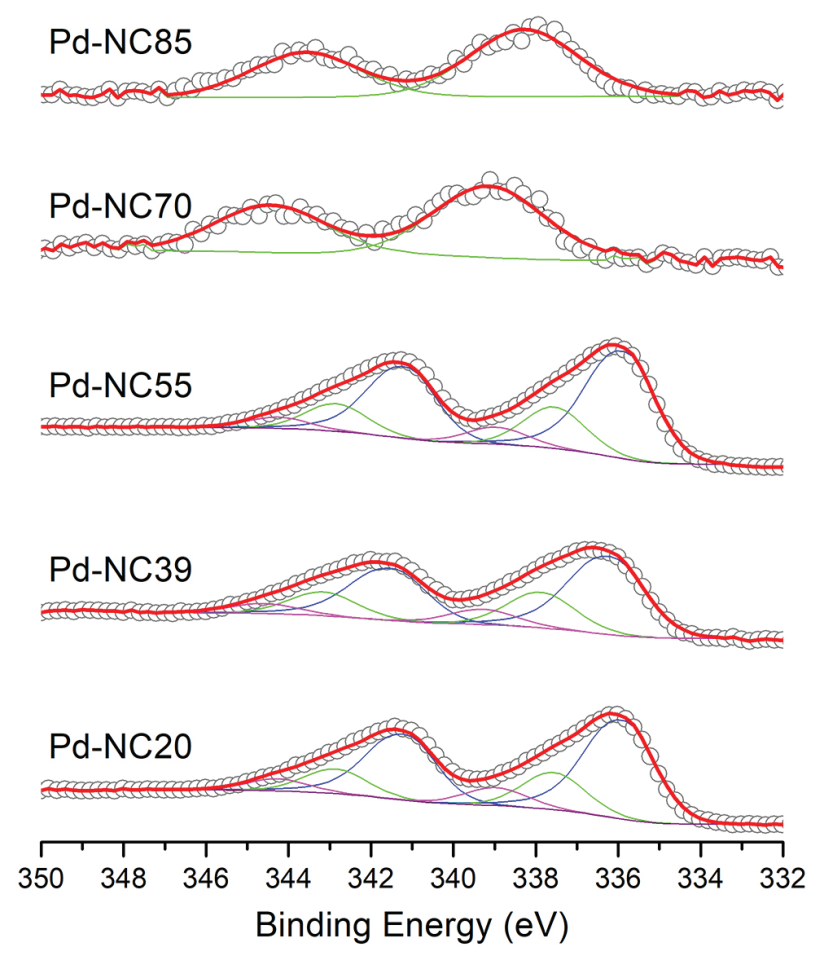

Figure 4. Deconvoluted XPS spectra of Pd 3d core level for the Pd-NCs with different amount $\left(\%, \mathrm{~m} \mathrm{~m}^{-1}\right)$ of PVP on the surface. suggest that Pd exposure simply increases; not a remarkable change on oxidation state or species when the PVP is removed was observed.

Table 1 also shows $\mathrm{N}, \mathrm{C}$ and $\mathrm{O}$ 1s regions for each sample. For the Pd-NC85 sample, N 1s peak is detected at $402.2 \mathrm{eV}$. N 1s from free PVP is observed at ca. $400 \mathrm{eV}$ ascribed to pyrrolidone group. ${ }^{17,18}$ At higher energies, $\mathrm{N} 1 \mathrm{~s}$ can be attributed to decreased electron density of the $\mathrm{N}$ group, when PVP chemisorb on metal surface through the $\mathrm{N}$ and $\mathrm{O}$ atom. Increasing the number of washes, the $\mathrm{N} 1 \mathrm{~s}$ peak position slowly approaches of free PVP $(399.9 \mathrm{eV}) .^{18}$ Comparing relative areas, it is clear that N/Pd ratio progressively changed upon treatment, which agrees with the removal of PVP. The signal-to-noise quality of $\mathrm{N} 1 \mathrm{~s}$ peak progressively decreases with the number of washes, contrary to Pd region of spectra. Here, changes in binding energies do not reveal any evolution on PVP in samples due to the wash procedure.

The XPS signal for $\mathrm{C} 1 \mathrm{~s}$ consisted of two components with BE at 287.6 and $291.1 \mathrm{eV}$ for Pd-NC20 sample, which can be assigned to aliphatic $\mathrm{C}_{\alpha}$ (lower BE) and the $\mathrm{C}^{\delta+}$ bound to the $\mathrm{N}$ and $\mathrm{O}$ atoms in PVP (higher BE).$^{17}$ While small changes in $\mathrm{C}_{\alpha} / \mathrm{C}^{\delta+}$ ratio (from 3.5 to $\mathrm{Pd}-\mathrm{NC} 85$ up to 6.7 to Pd-NC20) can be attributed to the wash procedure, no significative changes among samples on $\mathrm{BE}$ values were observed. The $\mathrm{O} 1 \mathrm{~s}$ spectra are overlaid with the $\mathrm{Pd} 3 \mathrm{p} 3 / 2$ peak at ca. $534 \mathrm{eV}$, so that limits further interpretation of O signals.

The characterization analysis described above shows that the nanoparticles purification through this simple washing off protocol is reliable and that the final Pd-NCs preserves its shape and surface nature, but a higher degree of agglomeration is present if the number of washing times is increased due to decrease of protective outer layer by removal of capping agent (PVP).

Table 1. Binding energies (BE) of the elements on the surface of the catalysts determined by XPS

\begin{tabular}{|c|c|c|c|c|c|c|}
\hline Sample & $\mathrm{Pd} 3 \mathrm{~d} 5 / 2 / \mathrm{eV}$ & $\mathrm{N} 1 \mathrm{~s} / \mathrm{eV}$ & $\mathrm{C} 1 \mathrm{~s} / \mathrm{eV}$ & $\mathrm{O} 1 \mathrm{~s} / \mathrm{eV}$ & $\mathrm{N} / \mathrm{Pd}$ ratio & $\mathrm{Pd}^{0} / \mathrm{Pd}^{\delta+}$ ratio \\
\hline Pd-NC85 & $338.3\left(\mathrm{Pd}^{\delta+}\right)$ & 402.2 & $\begin{array}{l}287.3 \\
289.8 \\
\end{array}$ & 534.2 & 19.8 & - \\
\hline Pd-NC70 & $339.1\left(\mathrm{Pd}^{\delta+}\right)$ & 402.8 & $\begin{array}{l}287.4 \\
290.3 \\
\end{array}$ & 534.7 & 9.0 & - \\
\hline Pd-NC55 & $\begin{array}{l}335.8\left(\mathrm{Pd}^{0}\right) \\
337.5\left(\mathrm{Pd}^{\delta+}\right) \\
338.9\left(\mathrm{Pd}^{\delta+}\right) \\
\end{array}$ & 401.7 & $\begin{array}{l}287.3 \\
289.7\end{array}$ & 534.4 & 4.7 & 2.1 \\
\hline Pd-NC39 & $\begin{array}{l}336.2\left(\mathrm{Pd}^{0}\right) \\
337.9\left(\mathrm{Pd}^{\delta+}\right) \\
339.3\left(\mathrm{Pd}^{\delta+}\right) \\
\end{array}$ & 400.7 & $\begin{array}{l}287.6 \\
291.1\end{array}$ & $\begin{array}{l}535.0 \\
532.4\end{array}$ & 2.5 & 1.8 \\
\hline Pd-NC20 & $\begin{array}{c}335.9\left(\mathrm{Pd}^{0}\right) \\
337.6\left(\mathrm{Pd}^{\delta+}\right) \\
339.0\left(\mathrm{Pd}^{\delta+}\right)\end{array}$ & 400.9 & $\begin{array}{l}287.6 \\
291.1\end{array}$ & $\begin{array}{l}535.2 \\
532.7\end{array}$ & 1.1 & 2.1 \\
\hline
\end{tabular}

XPS: X-ray photoelectron spectroscopy; $\mathrm{Pd}^{0}$ : metallic $\mathrm{Pd} ; \mathrm{Pd}^{\delta+}$ : oxidized Pd species. 
The influence of the amount of PVP in the outer layer of the Pd-NCs were evaluated in the catalysis for the hydrogenation of ST and PA, using THF as solvent. Mild reaction conditions ( 4 bar of $\mathrm{H}_{2}$ and $60{ }^{\circ} \mathrm{C}$ ) were found to be efficient to observe the hydrogenation activity. Figure 5 shows typical kinetic profiles for the substrate's consumptions. The catalytic activity of all Pd-NCs was assessed by following the progress of the reaction by $\mathrm{H}_{2}$ uptake (see Figures S3 and S4, SI section, for all kinetic profiles with ST and PA, respectively).

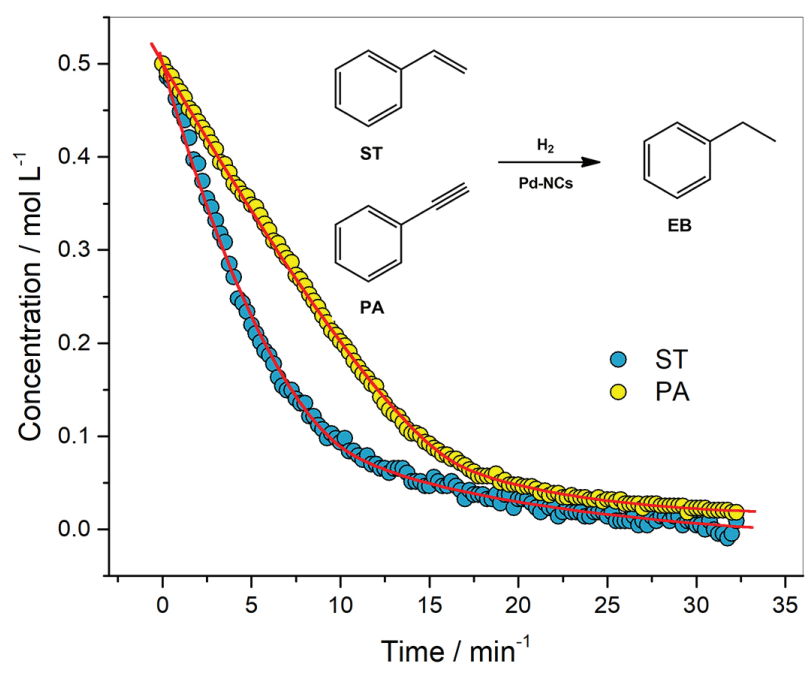

Figure 5. Time dependence of styrene (ST) and phenylacetylene (PA) concentration on the hydrogenation reaction catalyzed by Pd-NCs with $55 \%\left(\mathrm{~m} \mathrm{~m}^{-1}\right)$ of PVP on the surface.

In the PA hydrogenation, for all catalysts, no selectivity was observed in the triple bond reduction and ethylbenzene (EB) was the solo product (>99\%) observed by GC-MS at the full conversion of phenylacetylene. As well for ST hydrogenation, the formation of EB product was > 99\% at the full conversion of ST, except for Pd-NC85, where no reaction was observed. For the sake of comparison between the different catalysts, the rate constants for the $\mathrm{H}_{2}$ consumption were determined from a first-order kinetic analysis (Figures S3 and S4, SI section, for ST and PA hydrogenation, respectively).

The comparison of the rate constants for the Pd-NCs possessing different amount of PVP on the outer layer of the nanoparticles is shown on Figure 6. The profile presented in Figure 6 is a bell-like shape, with both reactions presenting the highest catalytic activity at $55 \%\left(\mathrm{~m} \mathrm{~m}^{-1}\right)$ of PVP. It is interesting to note that the difference in the rate constants between both substrates depends on the amount of PVP. Below 55\% of PVP, ST hydrogenation presents the higher rate constants when compared with PA hydrogenation, but this difference gradually decreases and at $20 \%$ of PVP non virtually difference between the rate constants is observed.

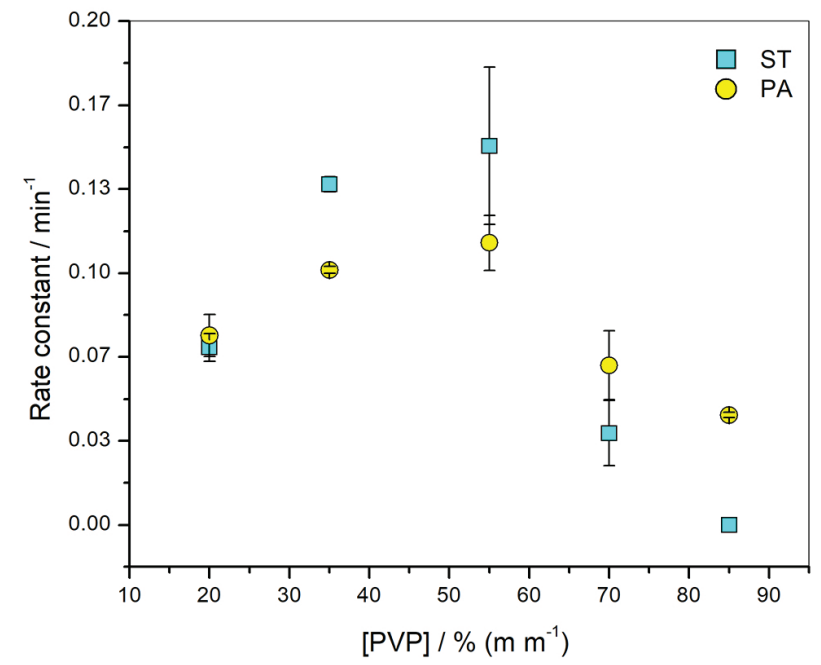

Figure 6. Reaction rate constant (k) dependence on the amount of PVP $\left(\mathrm{m} \mathrm{m}^{-1}\right)$ on the surface of Pd-NCs for styrene (ST) and phenylacetylene (PA) hydrogenation.

Above 55\% of PVP, the PA hydrogenation presents higher rate constants than ST hydrogenation. These behaviors show that the activity of the NCs can be modulated by the amount of PVP and that is affecting the different absorption capabilities of both substrates. Nonetheless, no selectivity of PA hydrogenation was observed, which means that there is no thermodynamic selectivity (i.e., when adsorption of alkyne >> adsorption of alkene). These results agree with the proposed mechanism involving the direct conversion of PA to EB, without any intermediate desorption/ re-adsorption of the ST. ${ }^{19}$ To understand the role of PVP on the reaction, it is necessary to rule out a possible diffusioncontrolled process of the reaction. Thus, the Damkohler number (DaII) for all catalysts were determined. The DaII is a useful ratio for determining whether diffusion rates or reaction rates are more important for defining a steadystate chemical distribution over the length and time scales of interest: ${ }^{20}$

$\mathrm{DaII}=\frac{\mathrm{kc}^{\mathrm{n}-1}}{\beta \mathrm{a}}$

where $\mathrm{k}$ is the reaction rate constant, $\mathrm{c}$ is the concentration, $\mathrm{n}$ is the reaction order, which is $\mathrm{n}=1$ by a first order reaction, $\beta$ is the mass transport coefficient and $a$ is the total area of the interface. The mass transport coefficient $\beta$ was determined by the ratio between the diffusion coefficient (D) of the substrates in solution, determined by diffusion-ordered nuclear magnetic resonance (NMR DOSY) experiments (Figure S5, SI section), and the characteristic length scale $\delta$ over which mass transport takes place, ${ }^{20}$ determined from the difference between the hydrodynamic diameter (from DLS analysis, Table S3, SI 
section) and the diameter of the metallic core, determined by TEM analysis.

Normally, for DaII $>>1$ the reaction rate is much greater than the diffusion rate and the reaction is said to be diffusion limited. On the other hand, a DaII $<<1$ points to a reaction rate-controlled system, where the diffusion is much faster compared to the reaction rate. In our case, the DaII magnitude was in the order of $10^{-5}$ to $10^{-6}$ (Table S3, SI section), these results indicate that the reaction rate is dominant over the diffusion rate. Nonetheless, the reaction is dependent on the amount of capping agent on the outer layer covering the Pd-NCs. Thus, we envision that PVP organization on the outer layer of the NCs, induced by the amount of PVP, may be responsible for the sequestration of the substrates from the solvent and increase the local concentration on the surface of the NCs and thus enhance the reaction rate. Substrate local concentration augment on nanoparticle's surface is one of the known effect of the capping agents. ${ }^{3,21}$

Using bulkier substrates for the hydrogenation, steric effect may also play an important role on reaction rate. The highest activity reported as turnover frequency (TOF), for STB and DPA hydrogenation, was for the one with the smallest amount of PVP on the surface (Pd-NC20), see Figure 7. Once more, no selectivity was observed and diphenylethane (DPE) was the solo product (>99\%) observed by GC-MS at the full conversion of substrates, except for Pd-NC85, where no reaction was observed for the STB substrate. Since hydrogenation of the alkyne (DPA) is faster than the alkene (STB) hydrogenation, it is reasonable to assume that the lack of selectivity in the triple bond reduction is due to a mechanism involving the direct conversion of DPA to DPE, without the intermediacy of STB and no thermodynamic selectivity is present. ${ }^{19}$

These results show that the balance between the catalytic activity of the NCs and the substrate structure can be modulated by the amount of capping agent present on the outer layer of the nanoparticles. This phenomenon is mainly attributed to steric, electronic and surface agglomeration effects, which include all surface phenomena directly related to the packing density, morphology and degree of order of the capping agent, which control the adsorption of the reactants and the isolation of the active site. The former is essentially determined by intermolecular interactions between the capping agent and the reactants, while the latter is influenced by geometric factors. ${ }^{21}$

\section{Conclusions}

In summary, we have shown that the simple protocol of washing off the excess of capping agent in catalytic

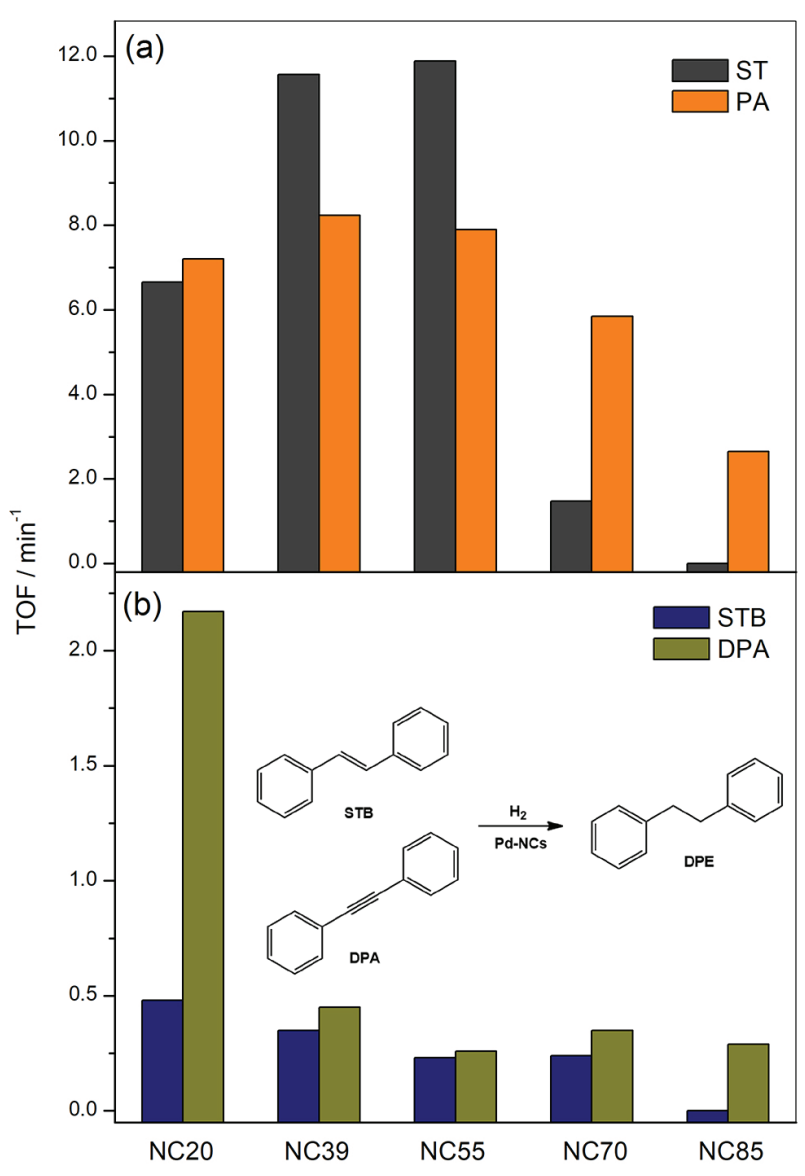

Figure 7. TOF as a function of Pd-NCs type for: (a) styrene (ST) and phenylacetylene (PA) and (b) stilbene (STB) and diphenylacetylene (DPA) hydrogenation reaction.

nanoparticles can directly influence the activity of the nanoparticles. Moreover, the results reported herein provide evidence that the activity of the nanoparticles is related to a balance between the amount of capping agent and the structure of the substrate, especially in terms of molecular bulkiness. Moreover, no selectivity in the triple bond reduction was observed, which means that the mechanism involves the direct conversion of the alkyne to alkyl molecules, without the intermediacy of the alkene.

\section{Supplementary Information}

Supplementary information (complementary spectroscopy and kinetic data) is available free of charge at http://jbcs.sbq.org.br as PDF file.

\section{Acknowledgments}

We are grateful to $\mathrm{CNPq}$ and CAPES for financial support received for this study. This work was also supported by the Brazilian Synchrotron Light Laboratory (LNLS) under proposal SAXS1-20170380. We also thank 
to LNLS for the XPS analysis, the Central Laboratory of Electron Microscopy (LCME) at UFSC and Prof Dr Cláudio F. Tormena from the Institute of Chemistry, UNICAMP, for the NMR diffusion experiments.

\section{References}

1. Koczkur, K. M.; Mourdikoudis, S.; Polavarapu, L.; Skrabalak, S. E.; Dalton Trans. 2015, 44, 17883.

2. Shen, J.; Ziaei-Azad, H.; Semagina, N.; J. Mol. Catal. A: Chem. 2014, 391, 36.

3. Niu, Z.; Li, Y.; Chem. Mater. 2014, 26, 72.

4. Lopez-Sanchez, J. A.; Dimitratos, N.; Hammond, C.; Brett, G. L.; Kesavan, L.; White, S.; Miedziak, P.; Tiruvalam, R.; Jenkins, R. L.; Carley, A. F.; Knight, D.; Kiely, C. J.; Hutchings, G. J.; Nat. Chem. 2011, 3, 551.

5. Naresh, N.; Wasim, F. G. S.; Ladewig, B. P.; Neergat, M.; J. Mater. Chem. A 2013, 1, 8553.

6. Safo, I. A.; Oezaslan, M.; Electrochim. Acta 2017, 241, 544.

7. Vidal-Iglesias, F. J.; Solla-Gullón, J.; Herrero, E.; Montiel, V.; Aldaz, A.; Feliu, J. M.; Electrochem. Commun. 2011, 13, 502.

8. Wang, H.; Tang, H.; He, J.; Wang, Q.; Mater. Res. Bull. 2009, 44,1676

9. Xian, J.; Hua, Q.; Jiang, Z.; Ma, Y.; Huang, W.; Langmuir 2012 , 28,6736

10. Elias, W. C.; Signori, A. M.; Zaramello, L.; Albuquerque, B. L.; de Oliveira, D. C.; Domingos, J. B.; ACS Catal. 2017, 7, 1462.
11. Cavalcanti, L. P.; Torriani, I. L.; Plivelic, T. S.; Oliveira, C. L. P.; Kellermann, G.; Neuenschwander, R.; Rev. Sci. Instrum. 2004, $75,4541$.

12. http://www.esrf.eu/computing/scientific/FIT2D, accessed in October 2019.

13. https://www.psi.ch/en/sinq/sansi/sasfit, accessed in October 2019.

14. Disch, S.; Wetterskog, E.; Hermann, R. P.; Wiedenmann, A.; Vainio, U.; Salazar-Alvarez, G.; Bergström, L.; Brückel, T.; New J. Phys. 2012, 14, 013025.

15. Disch, S.; Wetterskog, E.; Hermann, R. P.; Korolkov, D.; Busch, P.; Boesecke, P.; Lyon, O.; Vainio, U.; Salazar-Alvarez, G.; Bergström, L.; Brückel, T.; Nanoscale 2013, 5, 3969.

16. Sun, G.; An, J.; Hu, H.; Li, C.; Zuo, S.; Xia, H.; Catal. Sci. Technol. 2019, 9, 1238.

17. Zhong, R.-Y.; Sun, K.-Q.; Hong, Y.-C.; Xu, B.-Q.; ACS Catal. 2014, 4, 3982.

18. An, J.; Sun, G.; Xia, H.; ACS Sustainable Chem. Eng. 2019, 7, 6696.

19. Delgado, J. A.; Benkirane, O.; Claver, C.; Curulla-Ferré, D.; Godard, C.; Dalton Trans. 2017, 46, 12381.

20. Vannice, M. A.; Kinetics of Catalytic Reactions; Springer: New York, USA, 2005.

21. Campisi, S.; Schiavoni, M.; Chan-Thaw, C. E.; Villa, A.; Catalysts 2016, 6, 185.

Submitted: August 12, 2019 Published online: October 29, 2019 